\title{
Morphosyntax im deutsch-italienischen Vergleich: Eine kontrastive Fallstudie zu Wortbildungsprodukten in der internetbasierten Kommunikation der Online-Enzyklopädie Wikipedia
}

\begin{abstract}
Dieser Beitrag analysiert auf der Grundlage der Wikipedia-Korpora des Leibniz-Instituts für Deutsche Sprache morphosyntaktische Phänomene im deutsch-italienischen Vergleich. Konkret fokussiert die Fallstudie Konfixe, die ursprünglich lateinischen bzw. griechischen Ursprungs waren und zunächst überwiegend für den Bereich der Medizinfachsprache entlehnt wurden. Mittlerweile werden diese mit veränderter Semantik jedoch auch für gemeinsprachliche Wortbildungsprodukte eingesetzt: So finden sich - phob- (D) und -fob(IT) sowie -man- (D) und -man- (IT) in gemeinsprachlichen Wortbildungsprodukten, die formale und funktionale Äquivalenzen im Deutschen und Italienischen aufweisen. Wikipedia-Autor/-innen nutzen die als Krankheitsmetaphern zu deutenden Termini wie Lösch(o)manie oder cancellomania auf den Diskussionsseiten der Online-Enzyklopädie dazu, das Verhalten anderer Autor/-innen in der kollaborativen Textproduktion der Wikipedia metadiskursiv zu normieren.

This article analyses morphosyntactic phenomena in a comparison of German and Italian, based on the Wikipedia corpora of the Leibniz Institute for the German Language. Specifically, the case study focuses on confixes which were originally of Latin or Greek origin and were initially borrowed primarily in the field of medical terminology. With the passage of time, however, these have also come to be used, with changes to their semantics, in word formations in general usage. Thus - phob-(G) and -fob- (IT) as well as -man- (G) and -man- (IT) can be found in general usage in word formations which show formal and functional equivalences in German and Italian. Wikipedia authors use terms meant as disease metaphors such as Lösch(o)manie or cancellomania on the discussion pages of the online encyclopaedia in order to provide metadiscursive standardisation of the behaviour of other authors in the collaborative text production in Wikipedia.
\end{abstract}

\section{Einleitung ${ }^{1}$}

In ihrem Beitrag zum Konfix -phob- attestiert Trunkwalter dem Forschungsfeld zur Fremdwortbildung einen ,dringende[n] Forschungsbedarf, die Kombinationsmöglichkeiten von Fremdmorphemen, deren Funktionspotential und Gebrauchsradius detailliert zu analysieren“ (Trunkwalter 2009, S. 261). Die formulierte Notwendigkeit sieht sie insbesondere für Konfixe. Im Folgenden soll mit der Untersuchung der Konfixe -phob- (D) und -fob- (IT), -man- (D) und -man- (IT) im deutsch-italienischen Vergleich diese Forderung aufgegriffen werden. In der folgenden Analyse wird an der von Trunkwalter formulierten Trias möglicher Forschungsfoki aus Kombinationsmöglichkeiten, Funktionspotenzial und Gebrauchsradius weitergearbeitet. Insbesondere hinsichtlich des Gebrauchsradius macht der vorliegende Beitrag eine grundlegende Erweiterung gegenüber den Analysen zu -phob- von Trunkwalter (2009) und zu -man- von Feine (2009): Beide Analysen ziehen zur Untersuchung gemeinsprachlicher Wortbildungsprodukte mit den beiden genannten Konfixen Zeitungskorpora heran. In der folgenden Analyse wird das Spektrum des genutzten Datenmaterials auf Texte aus dem Bereich der internetbasierten Kommunikation konkret auf Benutzer-Diskussionsseiten der Online-Enzyklopädie Wikipedia - erweitert.

Die Autorinnen haben den Artikel gemeinsam konzipiert und bearbeitet. Eva Gredel hat die Abschnitte 2.1, 3.1, 3.2, sowie 4 und Carolina Flinz die Abschnitte 1, 2.2, 3.2 erarbeitet. 
Die vorliegende korpus-basierte Fallstudie ergänzt die bisherigen Analysen zu Konfixen bzw. Konfixderivaten somit um Erkenntnisse zu einer neuen, in diesem Forschungsfeld bisher wenig berücksichtigten Kommunikationsdomäne, die etwa in laienlinguistischen Diskursen zum angeblichen Sprachverfall intensiv und kontrovers verhandelt wird. Mit dem Beispiel der Wikipedia wurde dabei eine der ältesten und erfolgreichsten Plattformen des Social Web als Untersuchungsgegenstand ausgewählt. Zentral ist, dass sich die Diskussionsseiten der Wikipedia durch interaktionsorientiertes Schreiben (Storrer 2013, S. 337) auszeichnen, während in Zeitungstexten eher textorientierte Schreibformen auszumachen sind. Beim textorientierten Schreiben ,erwartet man eine Anpassung an Strukturierungsmuster und Formulierungstraditionen“ (ebd.), wobei dies beim interaktionsorientierten Schreiben nicht zum Erwartungshorizont der Beteiligten zählt. Wie sich dies auf die Wortbildung im skizzierten Bereich der Konfixderivate auf Diskussionsseiten in Wikipedia auswirkt, ist Gegenstand des Beitrags. Interaktionsorientierte Schreibformen mit ihren spezifischen Sprachmustern sind dabei längst keine Randphänomene mehr (vgl. ebd., S. 221), sondern prägen in Zeiten der Digitalisierung alltägliche Lebenswelten.

Analysiert wird, ob mithilfe der als „Europäismen“ (Trunkwalter 2009, S. 297) zu klassifizierenden Konfixe wikipedia-spezifische Determinativkomposita gebildet werden, die in der Werkstattsprache der deutschen wie auch der italienischen Sprachversion der Wikipedia Verbreitung gefunden haben und dort genutzt werden, um sich im Projekt der kollaborativen Textproduktion metakommunikativ zu artikulieren. Der Beitrag folgt mit diesem Interesse der textlinguistisch-pragmatischen Analyse von Konfixen nach Feine/ Habermann (2005, S. 89).

Das Corpus Search, Management and Analysis System COSMAS II ${ }^{2}$ bietet für dieses Vorhaben den empirischen Zugang über große Korpora, da dort neben den deutschsprachigen auch italienischsprachige Wikipedia-Daten zur Verfügung stehen. Um dem umfangreichen Material in beiden Sprachen gerecht zu werden, konzentriert sich dieser Beitrag auf die Betrachtung der Konfixderivate -phobie (D)/-fobia (IT) sowie -manie (D)/mania (IT) als Determinatum in (nominalen) Determinativkomposita. Die Analyse folgt dabei den folgenden Forschungsfragen:

a) Gebrauchsradius der analysierten Wortbildungsprodukte: Kommen gemeinsprachliche manie-/phobie-Kombinationen im Deutschen und mania-/fobia-Kombinationen im Italienischen in der internetbasierten Kommunikation der Wikipedia vor? Welche Vorkommenshäufigkeiten weisen diese Kombinationen auf?

b) Funktionspotenzial (textuell-pragmatische Perspektive): Gibt es unter den gemeinsprachlichen Kombinationen auf Wikipedia-Diskussionsseiten Kombinationen, die die kollaborative Text- und Wissenskonstruktion in Wikipedia metakommunikativ thematisieren? Gibt es wikipedia-spezifische Kombinationen?

c) Kombinationsmöglichkeiten (morphologisch-strukturelle Perspektive): Welche Struktur und Form weisen die Komposita mit den Konfixderivaten auf?

d) Semantische Perspektive: Welche semantischen Merkmale sind den Wortbildungsprodukten mit den Konfixderivaten zu eigen?

Der Beitrag schließt mit der kontrastiven Perspektive und ganz konkret mit dem Vergleich der fokussierten Wortbildungsprodukte im Deutschen und im Italienischen.

2 https://cosmas2.ids-mannheim.de/cosmas2-web (Stand: 21.2.2020). 


\section{Theoretischer Rahmen}

2.1 Morphologischer Status von - phob- und -man- im Deutschen

Lange Zeit war der Begriff Konfix ein umstrittener Terminus im Forschungsfeld der Lehnwortbildung (vgl. Donalies 2009). Mittlerweile ist der Begriff für die Beschreibung von Wortbildungsmitteln im Deutschen anerkannt und hat etwa Eingang in die Duden-Grammatik (Duden 2016, § 994) gefunden. Für die hier folgende korpuslinguistisch informierte Fallstudie von Wortbildungsprodukten im Deutschen ist die Definition von Trunkwalter passend:

Konfixe sind inhaltskonstituierende, produktive, gebundene, unmittelbar oder mittelbar basisfähige und/ oder kompositionsgliedfähige Wortbildungseinheiten, die über eine Positionsvariabilität verfügen können. (Trunkwalter 2009, S. 267)

Die beiden hier analysierten Einheiten - phob- und -man- werden in Anlehnung an Trunkwalter (2009) und Feine (2009) als exogene Konfixe verstanden. Beide Untersuchungen belegen korpuslinguistisch die hohe Produktivität und den hohen Grad der Integration beider Einheiten im Deutschen. So schreibt Trunkwalter zur etymologisch-diachronen Perspektive auf - phob-: „Die Integration des griechischstämmigen Konfixes in die deutsche Sprache hat zu Veränderungen auf sämtlichen formalen Ebenen des ursprünglichen Morphems geführt“" (Trunkwalter 2009, S. 266).

Das Konfix -phob- ordnet Trunkwalter (ebd., S. 261) den „Europäismen“ bzw. „Internationalismen" zu und schließt mit ihrer Definition an die Ausführungen von Kirkness an, der als „Maßstab des Europäischen das Vorkommen in mindestens vier anderen Sprachen neben Deutsch“ (Kirkness 2001, S. 121) versteht. Daraus lässt sich die hohe Relevanz von -phob- in Bereichen ableiten, in denen es um die internationale Verständigung geht:

Das Wissen, dass das Konfix - phob- sprachübergreifend produktiv ist, ermöglicht seinen schnellen und sicheren Gebrauch innerhalb internationaler Kommunikation. Geeignete Übersetzungsvarianten müssen nicht gesucht werden, sondern das Konfix -phob- kann unter Anpassung an das jeweilige Sprachsystem in Ad-hoc-Bildungen verwendet werden. (Trunkwalter 2009, S. 300)

Dies ist auch bei der Analyse des Sprachmaterials im multilingualen Projekt der Wikipedia relevant. Für - phob- ist die Produktivität sowohl in der Fachsprache als auch in der Gemeinsprache nachweislich vorhanden (vgl. ebd., S. 261). Aus einer semantischen Perspektive betrachtet gehen mit den gemeinsprachlichen phob-Kombinationen die zwei Bedeutungsnuancen ,Angst' und ,Abneigung' einher (vgl. ebd., S. 298 f.). Auf der ausdruckseitig-morphologischen Ebene ist für die folgende Fallstudie zum Konfixderivat -phobie interessant, dass es , in eine Reihe von Wortbildungen 2. oder höheren Grades wie Germanophobia [...eingeht]“ (ebd., S. 268).

Ähnlich wie - phob- ist auch das griechischstämmige -man- nicht nur in der Fachsprache, sondern auch in der Gemeinsprache sehr produktiv und hat dort mittlerweile eine lange Tradition: Bereits , in der zweiten Hälfte des 18. Jahrhunderts ist Anglomanie als übertriebene Liebe alles Englischen und dessen unkritische Nachahmung zu finden“ (Feine 2009, S. 320). Feine benennt den folgenden Grund für die hohe Produktivität: „Die Lexeme zeigen auch, dass insbesondere -manie als Wortbildungselement aus dem Bereich der Fachsprache in die Gemeinsprache übergegangen ist, weil man mit ihm offensichtlich Bedeutungsnuancen im konnotativen Bereich effektiv zum Ausdruck bringen kann" (ebd., S. 322). Für den semantischen Gehalt von -man- in gemeinsprachlichen Wortbildungen fasst sie Folgendes zusammen (ebd., S. 344): 
als ,eine übertriebene Vorliebe/ein übersteigertes Maß an Interesse für etwas oder jmdn. habend` das in Abhängigkeit von Kontextelementen gradiert sein kann zwischen geringfügiger bis maßloser Normüberschreitung und sich auch im konnotativen Bereich zwischen ernsthafter Kritik und scherzhafter Mahnung bewegt.

Für das hier fokussierte Konfixderivat -manie weisen Feine und Habermann ein hohes Kombinationspotenzial in Zeitungsartikeln nach: „Die Konstituente -manie verbindet sich mit unterschiedlichen Basen“ (Feine/Habermann 2005, S. 92). Neben verbalen Stämmen können auch Fremdwörter, Konfixe und Eigennamen als Basen fungieren. Auf semantischer Ebene führen sie zu -manie aus:

Allen Wortbildungen ist gemein, dass das durch das Basissubstantiv bzw. Basisverb evozierte Verhalten , in übertriebenem Maße' vorhanden ist. Den Wortbildungen kann eine pejorisierende Bedeutungskomponente zugeschrieben werden. (ebd.)

Im Rahmen der empirischen Analyse soll auch überprüft werden, inwieweit diese semantischen Aspekte auf Wikipedia-Material zutreffen.

\subsection{Morphologischer Status von - fob- und - man- im Italienischen}

Die in diesem Beitrag fokussierten Wortbildungsmittel -fob- und -man-, die im Italienischen neoklassische Komposita bilden, werden in der Literatur unterschiedlich benannt: Dem Terminus elemento formativo (Iacobini 2004, S. 70; Iacobini 2015, S. 1661) kommt ein neutraler Status zu. Er findet seine Entsprechung im englischen Terminus combining form bzw. im französischen formant. Migliorini (1963) prägt den Begriff affissoide 3 mit der positionsbezogenen Unterscheidung in prefissoide und suffissoide, die als Teilgruppe der Kompositionskonstituenten zu verstehen sind (vgl. ebd.). Scalise (1983) benennt die Wortbildungsmittel als semiparola. ${ }^{4}$ Auch in Standardwerken, die das italienische Lexikon untersuchen, variieren die verwendeten Termini zur Benennung der Einheit: Dardano (1978) nennt sie elementi di forma colta, Serianni (1988) elementi formativi scientifici und Tekavčić (1980) elementi formativi scientifici e tecnici di origine latina o greca (1980). Die Wörterbücher des Italienischen bevorzugen hingegen die Benennung elemento compositivo, wobei die Unterscheidung getroffen wird, ob diese am Anfang oder am Ende des Wortes (vgl. Iacobini 2004, S. 71) stehen.

Die Benennung ,Konfix‘ (Confisso), der wir in diesem Aufsatz folgen, ist auch im Italienischen zu finden: Sie wurde 1988 von Martinet zum ersten Mal genutzt und später auch von De Mauro (1999) im Grande Dizionario Italiano dell'Uso (GDI) verwendet (vgl. Iacobini 2004, S. 71). Wenn wir die Einträge -fobia und -mania im $\mathrm{GDI}^{5}$ untersuchen, wird

3 Die Benennung affissoide hat auch Eingang in die grammatische Terminologie zur Beschreibung anderer Sprachen gefunden (vgl. Bombi 1993, S. 162 f.).

4 Im Englischen benutzt Scalise (1983) den Begriff stem.

5 Die Beschreibung im GDI stellt eher eine Ausnahme dar, da die meisten Wörterbücher des Italienischen die Beschreibung von -mania und -fobia als zweites Element eines Kompositums bevorzugen (für -mania u. a. Dardano 1982, S. 1107; Devoto/Oli 2003, S. 1212; DISC 1997, S. 1477; Il Grande Dizionario Garzanti della Lingua Italiana 1987, S. 1101 und für -fobia Dardano 1982, S. 731; Devoto/Oli 2003, S. 835; DISC 1997, S. 979; Il Grande Dizionario Garzanti della Lingua Italiana 1987, S. 739). Zu ergänzen ist, dass Il Grande Dizionario Garzanti della Lingua Italiana 1987 eine zusätzliche Beschreibung für -fobia, -fobo, -mania, -mane hinzufügt, um auf Gemeinsamkeiten sowohl in ihrer Herkunft als auch in ihrer Verbreitung hinzuweisen. 
dies bestätigt: -fobia wird als fachsprachliches Konfix beschrieben (GDI 1999, S. 1), das aus dem Griechischen -phobía (vgl. phóbos ,timore') abgeleitet ist und dem die folgenden semantischen Merkmale zu eigen sind: Angst, Abneigung, krankhafte Abneigung, Antipathie, Intoleranz (paura, ripugnanza, avversione morbosa, antipatia, intolleranza vgl. agorafobia, anglofobia etc.). Für -mania wird ebenfalls auf die griechische Herkunft (-manía ,follia') verwiesen (ebd., S. 1144). Drei Bedeutungen werden dem Terminus zugeschrieben: 1. (in der Psychologie) im Sinne von einer morbiden und unkontrollierbaren Tendenz, bestimmte Aktionen durchzuführen (tendenza morbosa e irrefrenabile a compiere determinate azioni, vgl. cleptomania); 2. als abnorme Leidenschaft für etwas, (passione incontenibile per qualcosa vgl. bibliomania); 3. in der Pflanzenkunde als übermäßige Produktion von Organen durch einen Pflanzenorganismus (produzione smodata di organi da parte di un organismo vegetale, vgl. rizomania).

Trotz der unterschiedlichen Benennung im Italienischen können jedoch Überschneidungen bei den beschriebenen Eigenschaften festgestellt werden: Die Konstituenten sind gebunden; sie haben Ähnlichkeiten sowohl zu Affixen als auch zu freien Lexemen und exogene Herkunft (meistens griechische, aber auch lateinische Herkunft). Sie sind produktiv und weisen Positionsvariabiliät auf. Neben ihrer Verwendung in Fachsprachen haben sie über Ad-hoc-Bildungen im 18. Jahrhundert durch das Französische Eingang in die Gemeinsprache, später durch eine interlinguale Konvergenz von Lehnwortbildungen und Lehnübersetzungen (vgl. Bombi 1993, S. 160) gefunden.

Diese exogenen Elemente ${ }^{6}$ werden genutzt, um neoklassische Komposita zu bilden, die auch als Internationalismen definiert werden können, da sie mit der gleichen fachsprachlichen Bedeutung und einer fast identischen Form in unterschiedlichen Sprachen verwendet werden (vgl. Iacobini 2004, S. 69). Mit dem Aufschwung der Massenmedien haben diese Komposita auch Eingang in die Gemeinsprache gefunden und haben sich an die Sprachregeln der jeweiligen Sprache angepasst. Die Konsequenz für das Italienische war die Verbreitung von Komposita mit einem ,elemento formativo' und einem Wort (vgl. agriturismo) (vgl. ebd., S. 70).

Die neoklassischen Komposita haben weder die Eigenschaft eines für das Italienische typischen Kompositums noch eines Derivats. Das wird auch bei den mit -fob- und -mangebildeten Komposita deutlich, die eine besondere Reihung der Konstituenten aufweisen, die für das Italienische unüblich ist: nämlich Determinans + Determinatum. Weitere Merkmale der neoklassischen Komposita sind, dass sie abgeleitet werden und Verben bilden können. Dies sind Eigenschaften, die auf Komposita, die nur mit italienischen Elementen gebildet werden, normalerweise nicht zutreffen (vgl. Iacobini 2004, S. 80). Zudem weisen sie eine besondere Länge (mehrere Konfixe können kombiniert werden) und eine hohe Produktivität ${ }^{7}$ sowohl in der Fachsprache als auch in der Gemeinsprache auf. Kompositionsbildungen nach dem Muster Konfix + Lexem scheinen in der Gemeinsprache zu dominieren, gefolgt vom Muster Konfix + Konfix, während das Muster Lexem + Konfix

\footnotetext{
Es ist sehr schwierig, in der italienischen Sprache wegen ihrer starken Verbundenheit zu ihrer lateinischen Herkunft zwischen indogenen und exogenen Wörtern, zwischen suffigierter Bildung und Simplizia zu unterscheiden (vgl. Iacobini 2015, S. 1661).

7 Zum Begriff Produktivität vgl. Gaeta/Ricca (2015, S. 848) sowie Gaeta/Ricca (2006).
} 
weniger häufig vorkommt, aber eine höhere Homogenität in den Bedeutungen aufweist. Die Produktivität dieser dritten Möglichkeit (darunter werden auch die Bildungen mit -mania ${ }^{8}$ und -fobia einbezogen) kann im Laufe der Zeit variieren, da sie auch mit extralinguistischen Faktoren verbunden ist: Die hohe Produktivität der Komposita mit -mania und -fobia in den Wikipedia-Korpora kann mit der internationalen Verständigung, die dort zustande kommt, begründet werden.

\section{Korpuslinguistische Analyse der Wortbildungsprodukte}

\subsection{Datengrundlage der Korpusanalyse: Die Wikipedia-Korpora des Leibniz-} Instituts für Deutsche Sprache

Als Datengrundlage zur korpuslinguistischen Erhebung der Wortbildungsprodukte werden die Wikipedia-Korpora des Leibniz-Instituts für Deutsche Sprache herangezogen, die über das Corpus Search, Management and Analysis System (COSMAS II) verfügbar sind (vgl. Margaretha/Lüngen 2014). Der Aufbau der Wikipedia-Korpora, die über COSMAS II verfügbar sind, spiegelt die Struktur der Wikipedia wider: Im Namensraum der Artikeldiskussionsseiten geht es darum, die Inhalte der enzyklopädischen Artikelseiten zu verhandeln und auf den Benutzerdiskussionsseiten werden die Beiträge einzelner Autor/-innen verhandelt. Insbesondere die Texte in den Teilkorpora zu den verschiedenen Typen an Wikipedia-Diskussionsseiten (Artikeldiskussionen wdd17, Benutzerdiskussionen wud17 und Redundanzdiskussionen wrd17) sind dem Bereich der internetbasierten Kommunikation zuzurechnen (vgl. Lüngen/Kupietz 2017). Für die Analyse der hier fokussierten Wortbildungsprodukte wurden die jeweils aktuellsten Korpora zu den Artikel- und Benutzerdiskussionen herangezogen. Für die Analyse der deutschen Wortbildungsprodukte kommen somit das wdd17 mit seinen rund 349 Millionen Tokens und das wud17 mit rund 309 Millionen Tokens zur Anwendung. Für die italienische Sprachversion der Wikipedia wurden die ebenfalls über COSMAS II zugänglichen Korpora wdi15 (italienische Artikeldiskussionen) mit ca. 463 Millionen Tokens und wui (italienische Benutzerdiskussionen) mit knapp 50 Millionen Tokens verwendet. Zur Operationalisierung der korpuslinguistischen Rekonstruktion der oben beschriebenen Komposita über COSMAS II wurde der Suchstring mit dem Platzhalteoperator Asterisk (in Kombination dann also *manie bzw. *mania und *phobie bzw. *fobia) ausgewählt. Durch eine manuelle Analyse der Trefferlisten wurden irrelevante Wortgebilde sowie die medizinfachsprachlichen Termini aussortiert.

\subsection{Ergebnisse der WP-Analyse im Deutschen}

\subsection{1 -phobie im Deutschen}

Die Korpusanalyse mit dem Suchstring *phobie hat für das Wikipedia-Korpus zu den Diskussionsseiten (wdd17) insgesamt 4.035 Treffer und für das Korpus zu den Benutzerdiskussionsseiten (wud17) insgesamt 945 Treffer ergeben. Unter den 537 *phobie-Wortformen (wdd17) bzw. 239 *phobie-Wortformen (wud17) waren 226 (wdd17) bzw. 70 (wud17) fachsprachliche Wortformen. Die besonders geringe Zahl der fachsprachlichen Kombinationen im Korpus der Benutzerdiskussionsseiten lässt sich mit der Funktion dieses Seitentyps in der Online-Enzyklopädie Wikipedia begründen: In diesem Bereich der

8 In Iacobinis (2004, S. 83) Auflistung werden auch Komposita mit -mania benannt (vgl. congressomania). 
Wikipedia sind nur bedingt Diskussionen über enzyklopädische Inhalte üblich. Sehr viel zentraler sind für die Benutzerdiskussionsseiten Äußerungen zu den jeweiligen Autor/ -innen und deren Beiträgen zur kollaborativen Textproduktion. Die Zahl der hier besonders fokussierten gemeinsprachlichen *phobie-Kombinationen (im Folgenden: * phobie $_{\mathrm{GS}}{ }^{-}$ Kombinationen) war deutlich höher als die Zahl der fachsprachlichen (307 im wdd17 und 169 im wud17). Die Häufigkeiten der einzelnen Kombinationen in den Korpora war jedoch relativ gering - nur wenige Kombinationen kommen häufiger als fünfmal im Korpus vor. Von den $*$ phobie $_{\mathrm{GS}}$-Kombinationen können im Teilkorpus wdd17 insgesamt 35 als wikipedia-spezifisch und im Teilkorpus wud17 insgesamt 30 Treffer als wikipediaspezifisch eingeschätzt werden.

\begin{tabular}{|c|l|c|l|}
\hline & & wdd17 & wud17 \\
\hline$[\mathbf{1}]$ & *phobie-Treffer & 4.035 & 945 \\
\hline$[2]$ & *phobie-Wortformen & 537 & 239 \\
\hline$[3]$ & *phobie $_{\mathrm{FS}}$-Kombinationen & 226 & 70 \\
\hline$[4]$ & *phobie $_{\mathrm{GS}}$-Komvinationen & 307 & 169 \\
\hline$[\mathbf{5}]$ & davon wikipedia-spezifisch & 35 & 30 \\
\hline
\end{tabular}

Tab. 1: Ergebnisse der Korpusanalyse zum Suchstring *phobie

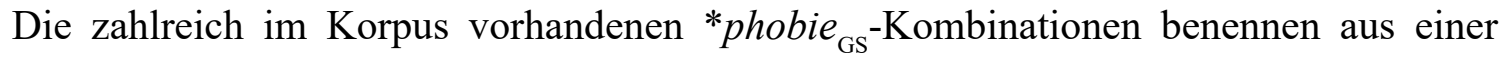
semantischen Perspektive die Angst vor oder Abneigung gegenüber Gegenständen (Knopfphobie, Erdbeerphobie) und Personen (Elvisphobie und Zahnarztphobie). Die morpholo-

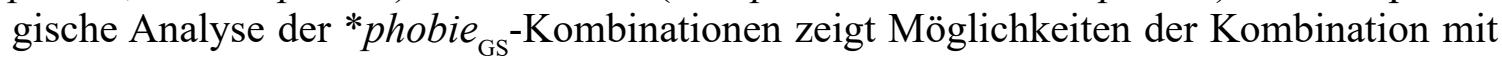
unterschiedlichen Konstituenten - darunter etwa Kurzwörter (,SPOV"-Phobie, AfD-Phobie und BK-Phobie) und Propria (z. B. Heilbronn in Heilbronnphobie). Bei -phob-gibt es gemeinsprachliche Wortbildungsprodukte, die mit der Fuge -o-gebildet werden (Troll-ophobie), und es finden sich auch Dubletten, die mit und ohne Fuge gebildet werden (Admin-o-phobie versus Adminphobie). Graphische Varianten mit und ohne Bindestrich sind ebenso vorhanden: Listenphobie versus Listen-Phobie, Rotlink-Phobie sowie Rotlinkphobie und Lateinphobie sowie Latein-Phobie.

Aus einer textuell-pragmatischen Perspektive lassen sich zahlreiche * phobie $e_{\mathrm{GS}}-\mathrm{Kombina}_{\mathrm{-}}$ tionen finden, denen die Funktion zukommt, in metakommunikativen bzw. metasprachliche Aussagen die kollaborative Textproduktion zu thematisieren. So geht es etwa bei den metasprachlichen * phobie ${ }_{\mathrm{GS}}$-Kombinationen um Angst vor dem falschen oder übermäBigen Einsatz von Interpunktionszeichen oder Elementen anderer Sprachen, wie das folgende Beispiel zeigt:

Man kann [...] die Denglisch-Phobie auch übertreiben. In unseren Medien ist es ja z. B. auch üblich geworden, US-Dienstgrade wie „Master Sergeant“ in meist überhaupt nicht adäquate deutsche Übersetzungen zu verbiegen. (Wikipedia 2009: Diskussion zu Waffenfarbe)

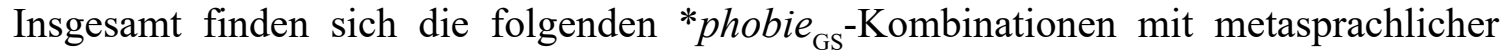
Funktion in den Wikipedia-Korpora:

Apostrophphobie (1), Bindestrichphobie (1), Denglisch-Phobie (1), Fremdwortphobie (1), LeerzeichenPhobie (1), minuskelphobie (1), Umlaut-Phobie (1), umlautphobie (1), Welche-Phobie (1) 
Die Zahl der * phobie $_{\mathrm{GS}}$-Kombinationen mit metakommunikativer Funktion ist noch sehr viel größer. Zum Teil werden hier sehr spezifische Aspekte der Kommunikation im Projekt der kollaborativ erarbeiteten Online-Enzyklopädie Wikipedia thematisiert:

„SPOV“-Phobie (2), Admin-Phobie (1), Adminophobie (1), Adminphobie (2), Anglizismenphobie (1), „Bürokratie“-Phobie (1), Bapperlphobie (1), Baustein-Phobie (2), Bausteinphobie (1), Belege-Phobie (1), Belegphobie (1), BKPhobie (1), Bkphobie (1), Datumslinkphobie (1), Hilfe\#IP-Phobie (1), IP-Phobie (3), Kategorienphobie „Bürokratie“-Phobie (1), Kompromissphobie (1), Listen-Phobie (1), Listenphobie (2), Löschphobie (1), MB-Phobie (1), Metadatenphobie (1), Namensraumphobie (1), Quellenphobie (1), Redundanz-Phobie (1), Referenzierungsphobie (1), Rot-Link-Phobie (1), rotlink-phobie (1), rotlinkphobie (1), Rotlink-Phobie (1), Rotlinkphobie, Scrollphobie (1), Sockenphobie (1), Sockenpuppenphobie (1), Spaltenphobie (1), Theoriefindungsphobie (1), Trollophobie (2), Vorlagenphobie (1), Wikipediaphobie (1), Wikiphobie (1)

Wörter wie etwa Socke(n) (in Sockenphobie) oder Sockenpuppe(n) (in Sockenpuppenphobie) sind in der Werkstattsprache der Wikipedia hochfrequent. Konkret bezeichnet Socke/ Sockenpuppe Wikipedia-Autor/-innen, die - entgegen der wikipedia-spezifischen Richtlinien - in der kollaborativen Textproduktion mit zwei Nutzerkonten agieren, um ihre präferierten Textteile bzw. Sichtweisen in der Online-Enzyklopädie durchzusetzen. In die-

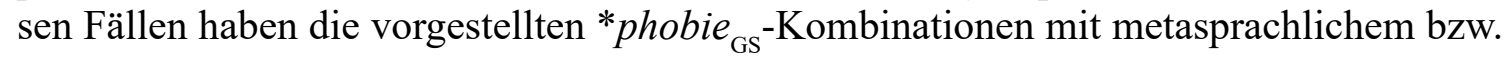
metakommunikativem Gehalt die Funktion, die kollaborative Textproduktion in der Wikipedia zu normieren und sie den Richtlinien der Wikipedia-Gemeinschaft anzupassen.

\subsection{2 -manie im Deutschen}

Die Korpusanalyse mit dem Suchstring *manie hat für das Wikipedia-Korpus zu den Diskussionsseiten (wdd17) insgesamt 967 manie-Treffer und für das Korpus zu den Benutzerdiskussionsseiten (wud17) insgesamt 470 Treffer ergeben. Unter den 254 (wdd17) bzw. 146 * manie-Wortformen (wud17) waren 27 bzw. 12 falsche Treffer, sodass nur 227 bzw. 134 Wortformen der Analyse zugeführt werden konnten. Fachsprachliche Kombinationen (im Folgenden: * manie $_{\mathrm{FS}}$-Kombinationen) sind in der Minderzahl in beiden Korpora (wdd17: 37 Kombinationen und wud17: 29 Kombinationen) - kamen aber mit relativ hohen Vorkommenshäufigkeiten vor (z. B. Nymphomanie mit 42 Treffern). Die Zahl der verschiedenen * manie $_{\mathrm{GS}}$-Kombinationen war höher (190 im wdd17 und $117 \mathrm{im}$ wud17), allerdings waren ihre Häufigkeiten in den meisten Fällen relativ gering: 167 der * manie $_{\mathrm{GS}}{ }^{-}$

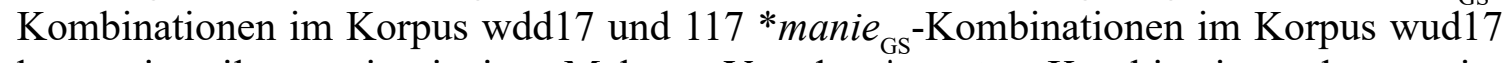

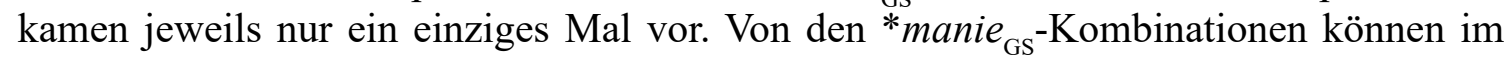
wdd17 insgesamt 79 als wikipedia-spezifisch und im wud17 insgesamt 47 als wikipediaspezifisch eingeschätzt werden.

\begin{tabular}{|c|c|c|c|}
\hline & & wdd17 & wud17 \\
\hline [1] & *manie-Treffer & 967 & 470 \\
\hline [2] & *manie-Wortformen & 227 & 134 \\
\hline [3] & ${ }^{*}$ manie $_{\mathrm{FS}}-$ Kombinationen & 37 & 29 \\
\hline [4] & ${ }^{*}$ manie $_{\mathrm{GS}}$-Kombinationen & 190 & 117 \\
\hline [5] & davon wikipedia-spezifisch & 79 & 47 \\
\hline
\end{tabular}

Tab. 2: Ergebnisse der Analyse zum Suchstring *manie 
Wenige * manie $_{\mathrm{GS}}$-Bildungen kommen häufiger als fünfmal im Korpus wdd 17 vor: Tulpenmanie (49), ${ }^{9}$ Keltomanie (42), Anglomanie (16), Bibliomanie (9), Listomanie (9), Löschmanie (9), Dinomanie (7), Germanomanie (6). Die folgenden *manie ${ }_{\mathrm{GS}}$-Bildungen kommen häufiger als fünf Mal im Korpus wud17 vor: Löschmanie (12), Tulpenmanie (20), Wikimanie (12), Keltomanie (8).

Die morphologische Analyse des Materials zeigt eine sehr große strukturelle Vielfalt: Bei den vorangestellten Determinans-Konstituenten kann es sich nicht nur um Zahlwörter (16:9-Manie), sondern auch um Kurzwörter (LA-Manie, RNA-Manie, PC-Manie, SMSManie, VS-Manie und Wiki-Manie) oder um Kurzwort-Komposita (etwa RFID-Pass-Verseuchungs-Manie) sowie Eigennamen (Henriette-Sontag-Manie, Obama-Manie, PotterManie) handeln. Die Determinans-Konstituenten sind bei den als fachsprachlich zu klassifizierenden Manie-Komposita ausnahmslos exogen (z. B. Kleptomanie). Bei den gemeinsprachlichen klassifizierten Komposita sind zudem auch zahlreiche indogene Konstituenten vorhanden, z. B. Eichenmanie, Farbmanie, Namensmanie, Oberlehrermanie und Quellenmanie.

Die Analyse des Materials zeigt, dass dem Konfixderivat -manie bis zu 20 Glieder in der internetbasierten Kommunikation der deutschen Wikipedia vorangestellt sind, die nicht nur komplexere Wortbildungsprodukte, sondern z. T. auch Äquivalente zu HauptsatzNebensatz-Konstruktionen darstellen.

a) ,ich-mach-einen-Kasten-in-den-Artikel-auch-wenn-ich-keine-Ahnung-hab-wie-ichmit-den-Fotos-umzugehen-habe“-Manie;

b) „Was-ich-privat-denke-muss-unbedingt-hier-ausgewalzt-werden“-Manie,

c) Reim-dich-oder-ich-beiss-dich-Manie

d) Unter-Unter-Unter-Genre-Manie.

Bei Wortbildungsprodukten mit exogenen Determinata (Bibli-o-manie, Gigant-o-manie, Graph-o-manie) sowie bei Kombinationen mit Nationalitäten (Ägypt-o-manie, Celt-omanie, German-o-manie, Graec-o-manie) kommt das Fugenelement -o- vor. Auch in gemeinsprachlichen Kombinationen finden sich Wortbildungsprodukte mit dem Fugenelement -o- (z. B. Rank-o-manie, Revert-o-manie, Druid-o-manie, Erot-o-manie, Legendo-manie, Myth-o-manie, Papyr-o-manie, Twist-o-manie). Dubletten sind bei der Bildung gemeinsprachlicher Wortbildungsprodukte ebenso vorhanden:

\begin{tabular}{|l|l|}
\hline mit Fuge -o- & ohne Fuge -o- \\
\hline List-o-manie & Listen-Ø-manie \\
\hline Link-o-manie & Link-Ø-Manie \\
\hline Lösch-o-manie & Lösch-Ø-manie \\
\hline Quell-o-manie & Quellemanie/Quellenmanie \\
\hline Revert-o-manie & Revert-Ø-manie \\
\hline Tulp-o-manie & Tulpen-Ø-manie \\
\hline
\end{tabular}

Tab. 3: Beispiele für Dubletten mit und ohne Fuge

\footnotetext{
9 Hier und im Folgenden werden in Klammern die absoluten Vorkommenshäufigkeiten angegeben.
} 
Die Mehrzahl der gemeinsprachlichen Kombinationen wird jedoch ohne die Fuge -ogebildet. Auch graphische Varianten mit und ohne Bindestrich bei der Schreibung sind vorhanden (Bausteinmanie versus Baustein-Manie, Löschmanie versus Lösch-Manie sowie Katmanie versus Kat-Manie). Der semantische Gehalt der *manie ${ }_{\mathrm{GS}}$-Kombinationen umfasst eine übertriebene Vorliebe für bestimmte Objekte (Tulpenmanie), Personen (Aristoteles-Manie) oder Prozesse/Tätigkeiten (Korrigiermanie). Metakommunikative und metasprachliche ${ }^{*}$ manie $_{\mathrm{GS}}$-Kombinationen sind sehr häufig und $\mathrm{z}$.T. wikipedia-spezifisch. Mithilfe der metakommunikativen Kombinationen werden vielfältige Aspekte der kollaborativen Textproduktion in der Online-Enzyklopädie Wikipedia thematisiert:

„Quellen“-Beleg-Manie (1), Auslagerungsmanie (2), Bausteinmanie (2), Bebilderungsmanie (1), Bildverbreitungsmanie (3), Botmanie (1) edit-revert-Manie (1), Editcount-Manie (1) Enzyklopädieverbesserungsmanie (1), hauptautorenmanie (1), Kartenmanie (1), Kategorierungsmanie (2), Kategorisierungsmanie (1), Link-Manie (1), Linkomanie (3), Listenmanie (5), Listomanie (9), Löschantrags-Manie (2), Löschmanie (9), Löschomanie (1), löschomanie (1), Löschungsmanie (1), Nachweismanie (1), Quellemanie (1), Quellenangabe-Manie (1), Quellenmanie (2), Quellomanie (5), redundanzvermeidungsmanie (1), Referenzierungsmanie (2), Revertmanie (1), Revertomanie (2), Rotlink-Manie (1), Schnelllöschmanie (1), Sockenpuppen-Manie (1), Tabellenmanie (1), Übersetzungs-Manie (1), Übersetzungsmanie (1), Unter-Unter-Unter-Genre-Manie (1), Verlinkungsmanie (2) verlinkungsmanie (3), wikipedia-schreibweisenmanie (1), Zitatmanie (2)

Die im engeren Sinne metasprachlichen Aspekte thematisieren den Umgang mit Sprache bei der Erstellung von Wikipedia-Artikeln.

„Jänner“-Manie (1), Jänner-Manie (1),,auch“-Manie (1), auch-Manie (1), Abkürzungsmanie (4), Anglizismusmanie (1), Binde-Strich-Setzung-S-Manie (1), Fachausdrucksmanie (1), Kleinschreibmanie (1), Leerzeichenmanie (1), Paraphrasierungsmanie (2), Personalpronomen-Manie (1), Schreibmanie (1), Siehe-Auch-Manie (1)

Die Bedeutung aller gemeinsprachlichen Kombinationen zeichnen die Seme ,eine übertriebene Vorliebe habend' bzw. ,ein übersteigertes Maß an Interesse habend‘ aus, wie etwa der Beleg unter der Überschrift Löschomanie zeigt:

\section{e) Löschomanie}

[...] Es passiert leider zu oft, dass schlechte Artikel gelöscht werden müssen. Sie MÜSSEN auch gar nicht gelöscht werden; mensch könnte sie auch verbessern. (Wikipedia 2014: Diskussion zu Nexialismus)

Die vorgestellten Ergebnisse der Korpusstudie belegen die hohe Produktivität von -manbzw. -manie in der internetbasierten Kommunikation der deutschen Wikipedia-Sprachversion.

\subsection{Ergebnisse der Wikipedia-Analyse im Italienischen}

\subsection{1 -fobia im Italienischen}

Die Korpusanalyse im Wikipedia-Korpus mit dem Suchstring *fobia hat insgesamt 1.044 fobia-Treffer zu den Diskussionsseiten (wdi15) und 424 Treffer zu den Benutzerdiskussionen (wui15) ergeben: 


\begin{tabular}{|c|c|c|c|}
\hline & Korpus & wdi15 & wuil5 \\
\hline [1] & *fobia-Treffer & 1.044 & 424 \\
\hline [2] & *fobia-Wortformen & 136 & 112 \\
\hline [3] & ${ }^{*}$ obia $_{\mathrm{FS}}-$ Kombinationen & 23 & 22 \\
\hline [4] & ${ }^{*}$ fobia $_{\mathrm{GS}}-$ Kombinationen & 49 & 32 \\
\hline [5] & davon wikipedia-spezifische $*$ fobia $_{\mathrm{GS}}-$ Kombinationen & 3 & 7 \\
\hline
\end{tabular}

Tab. 4: Ergebnisse der Suchanfrage mit dem Suchstring *fobia

Unter den fobia-Wortformen (136 im wdi15 und 112 in wui15) wurden die irrelevanten oder fehlerhaften Wortgebilde (wie u. a. all'omofobia, cancellare/Italofobia, dall'eterofobia, Uderzo-critiche-xenofobia etc.) nicht berücksichtigt. Die daraus resultierende Materialbasis bezeugte in beiden Korpora eine größere Häufigkeit an gemeinsprachlichen gegenüber fachsprachlichen Kombinationen, die meistens Ad-hoc-Bildungen (26) sind, wie u.a. afghanofobia, arabofobia, calciofobia, cattolicofobia, cultofobia, drogofobia, silviofobia, singlefobia. Für eine kleinere Gruppe (11) wurden weniger als fünf Belege gefunden (agorafobia, ${ }^{10}$ claustrofobia, cristianofobia, eterofobia, francofobia, giudeofobia, islamofobia, italofobia, omofobia, transfobia, xenofobia), während es für die restlichen mehr als fünf Belege waren. Hohe Vorkommenshäufigkeiten zeigen insbesondere die folgenden Bildungen: islamofobia (39), omofobia (232), sessuofobia (91), xenofobia (187). Insgesamt gab es neun als wikipedia-spefizisch einzuschätzende Wortbildungsprodukte: Im wdi15 waren dies nur metakommunikative Bildungen wie logicofobia (1), povfobia (1) und promofobia (1). Im Korpus wuils waren es sowohl metakommunikative Formen wie agorafobia (1), bloccofobia (1), bocciofobia (3), claustrofobia (2), idrofobia (1), puntofobia (1), templatefobia (2) als auch metasprachliche wie anglofobia (1), esterofobia (2).

Die morphologische Analyse der Datenbasis zeigt eine strukturelle Vielfalt: Erstglieder sind meistens exogen (agorafobia, singlefobia, templatefobia etc.); es kommen sowohl selbstständige als auch unselbstständige Konstituenten vor. Unter den selbstständigen finden sich Substantive (wie tabaccofobia und clerofobia), Adjektive (cristianofobia und cattolicofobia) sowie Verben (bloccofobia, bocciofobia). Unter den unselbstständigen finden sich die meisten weiteren Konfixe wie antiomofomia, transfobia und xenofobia. Ein Wortbildungsprodukt mit einem Kurzwort als erster Konstituente kommt ebenfalls vor (pov-fobia). Konstituenten, die äquivalent zu Sätzen und Zahlen sind, sind nicht im Korpus vorhanden. Die Endung der ersten Konstituente des Kompositums auf -o kann mit der typischen Uniformierungstendenz der neoklassischen Komposita in Verbindung gebracht werden: Wenn das Konfix (bzw. Konfixderivat), das die zweite Einheit des Kompositums bildet, griechischer Herkunft ist, wird die Endung der ersten Konstituente hin zu -o modifiziert bzw. ein -o ergänzt (u. a. islam-o-fobia). ${ }^{11}$ Graphische Varianten mit und ohne Bin-

10 Bei einigen Komposita ist sowohl ein fachsprachlicher als auch ein gemeinsprachlicher Gebrauch festzustellen, z. B. agorafobia und claustrofobia.

11 Wenn die erste Konstituente des Kompositums schon mit -o endet, findet keine Änderung statt; wenn das erste Wort mit einem anderen Vokal endet, findet die Modifizierung statt; wenn das erste Wort mit einem Konsonanten endet, wird der Vokal -o hinzugefügt (vgl. Iacobini 2004, S. 76 f.). 
destrich sind ebenso vorhanden: anti-omofobia versus antiomofobia. In seltenen Fällen (anti-omofobia, Hexakosioihexekontahexafobia, JacopoWertherfobia, non-omofobia, omotransfobia, sesquipedalofobia) überschreiten die Wortbildungen zwei Glieder.

Die vorangestellten Determinans-Konstituente sind entweder Eigennamen (JacopoWerther, silvio, wimbfobia), Orte (Pescinofobia), ethnische Herkunftsangaben (afghanofobia, anglofobia, arabofobia, croatofobia etc.), Charakteristika der Person, die mit Glauben, sexueller Haltung, physischen Eigenschaften verbunden sind (cattolicofobia, eterofobia, sordofobia etc.), Gegenstände (telefoninofobia), Aktivitäten (calciofobia), Tiere (lumaco-fobia) und Blumen (agavofobia).

In den gemeinsprachlichen Wortbildungen können sowohl die Bedeutungsnuancen ,Angst' (agorafobia, claustrofobia, lumaco-fobia) als auch ,Abneigung' (francofobia, giudeofobia) rekonstituiert werden. Wortbildungsprodukte zur kollaborativen Textproduktion enthalten ebenfalls beide Bedeutungen: ,Angst' wie u. a. in agorafobia, claustrofobia, bloccofobia und ,Abneigung', wie u. a. in puntofobia, anglofobia.

(1) Wow! davvero interessante, ci darò sicuramente un'occhiata approfondita, anche se le voci preferisco scriverle su wikipedia ;-). Mi sale uno strano senso di agorafobia quando visito gli altri wikiprogetti $\mathrm{xD}$, tipo wikibooks o wikiversità... è già tanto se carico immagini su commons (Wikipedia 2013: Benutzerdiskussion zu Riccardo Rovinetti) ${ }^{12}$

(2) Gli spazi HTML che ho messo (nbsp;) servono a rendere la tabella stessa un pò più gradevole esteticamente: senza quelli, cifre e bordi stanno tutti appiccicati e mi danno una sensazione di claustrofobia: (se Utente vuole proprio fare altrimenti vabbè, ma se no preferirei che ci restino :) (Wikipedia 2004: Diskussion zu Funzione die ripartizione della variabile casuale normale) $)^{13}$

In (1) wird z. B. der zur Verfügung gestellte Platz für die redaktionelle Arbeit bei vielen Wiki-Projekten (agorafobia) thematisiert oder in (2) die Spaltengröße der Tabellen, die ohne Leerstellen eine zu komprimierte Darstellung verursacht (claustrofobia).

\subsection{2 -mania im Italienischen}

Die Suchanfrage mit dem Platzhalteroperator * in Kombination mit mania (*mania) hat insgesamt 188 (wdi15) und 219 (wui15) Wortformen, die auf -mania enden (6.010 Treffer in wdi15 und 4.858 Treffer in wui15), ergeben. Da die Treffer sowohl mania $_{\mathrm{FS}}-$ Kombi- $^{-}$ nationen als auch mania $_{\mathrm{GS}}$-Kombinationen enthalten, sowie irrelevante Wortgebilde wie Eigennamen von Autoren (Baustellemania), von Zeichentrickfilmen (Tazmania) oder Fremdwörter (trichotillomania) oder sonstige Wortformationen (dall'ABBAmania), sind die Daten manuell ausgewertet worden. Die Ergebnisse sind in folgender Tabelle zusammengefasst:

12 Übersetzung (CF): Wow! Wirklich interessant, ich werde da sicherlich ein Auge darauf werfen, auch wenn ich die Einträge in Wikipedia zu schreiben bevorzuge :) es steigt in mir ein komisches Gefühl von Agoraphobie auf, wenn ich die anderen Wikiprojekte, wie Wikibooks oder Wikiversità öffne...es ist schon viel, wenn ich die Bilder auf Commons hochlade.

13 Übersetzung (CF): Die HTML-Leerstellen, die ich eingefügt habe, lassen die Tabelle ästhetischer erscheinen: Ohne sie sind Zahlen und Ränder ganz eng und geben mir ein Gefühl von Klaustrophobie. 


\begin{tabular}{|c|c|c|c|}
\hline & Korpus & wdi15 & wui15 \\
\hline [1] & *mania-Treffer & 6.010 & 4.858 \\
\hline [2] & *mania-Wortformen & 188 & 219 \\
\hline [3] & * mania $_{\mathrm{FS}}-$ Kombinationen & 11 & 8 \\
\hline [4] & * ania $_{\mathrm{GS}}$-Kombinationen & 55 & 52 \\
\hline$[5]$ & davon wikipedia-spezifische $*{ }^{*}{ }^{2} a n i a_{\mathrm{GS}}-$ Kombinationen & 6 & 7 \\
\hline
\end{tabular}

Tab. 5: Ergebnisse der Analyse zum Suchstring *mania

Von den mania $_{\mathrm{GS}}$-Bildungen in wdi15 und wui15 treten folgende Lexeme mehr als fünfmal auf: wikimania (1331), wrestlemania (61), megalomania ${ }^{14}$ (46), Beatlemania (10), grafomania (13), cancellomania (9), testimania (6). Die morphologische Analyse des

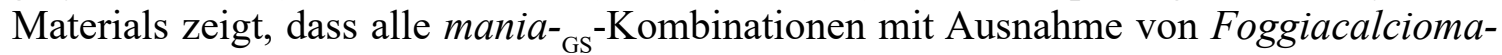
nia, wiknippomania und teuto-grafomania zweigliedrige Komposita sind. Viele Erstglieder sind exogen (anglomania, chatmania, consolemania, divemania, grafomania, ipodmania etc.). Die einzelnen Glieder sind meistens Simplizia, die mit Ausnahme von ricettemania, navimania, testimania und motorimania im Singular in die Kombinationen eingebunden sind. Kurzwörter, komplexe Wortbildungsprodukte, Satzkonstituenten und Zahlen sind nicht in den mania $_{\mathrm{GS}}$-Kombinationen vorhanden. In einem Fall wird ein substantiviertes Verb (,lesen`) als Erstglied benutzt (leggeremania) oder das Verb ,löschen“ (cancellomania). Wortbildungen nach dem Muster Konfix + Konfix sind ebenfalls in den Korpora vertreten, wie die mania-Bildungen mit Völkernamen/Ethnien als Erstglied bezeugen (anglomania, gallomania, illiro-mania, italomania, liguro-mania etc.).

Sowohl in den mania-Bildungen der Fachsprache (cocainomania, eroinomania etc.) als auch in denen der Gemeinsprache werden Lexeme mit der oben beschriebenen Uniformierungstendenz (vgl. Abschn. 3.3.1) folgend gebildet (u. a. parallelomania und tulipomania). In einem Fall wurde auch eine Dublette bemerkt (tulipanomania versus tulipomania): Im ersten Lexem endet die erste Konstituente schon mit $-o$, im zweiten wird der Vokal hinzugefügt. Die Endung der ersten Konstituente mit -i-, die die lateinische Herkunft hervorhebt, kommt hingegen nicht vor. Graphische Varianten bezüglich der Zusammenschreibung der Konstituenten (mit und ohne Bindestrich) sind nur in fachsprachlichen Komposita (ninfo-mania versus ninfomania und tricotillo-mania versus tricotillomania) vorhanden.

Semantisch auffällig ist, dass die Kombinationen entweder eine Vorliebe für etwas (Musik, Fußball, Sex) oder Maßlosigkeit ausdrücken. Eine semantische Verschiebung von der medizin-fachsprachlichen Bedeutung (,psychische Störung', ,Raserei', ,Wahnsinn') zu einer gemeinsprachlichen Bedeutung ,krankhaft', ,übertriebene Neigung', ,abnorme Leidenschaft' oder , eine übertriebene große Vorliebe/Leidenschaft für etwas habend' kommt deutlich hervor. Der Fokus der Seme, die in der Fachsprache auf ,krank', ,abnorm‘, ,Beeinträchtigung physischer Funktionen' lag, hat sich auf ,krankhaft', ,übertriebene Neigung' und ,abnorme Leidenschaft' verschoben. In den wikipedia-spezifischen Kombinationen kann nur die Variante ,krankhaft‘, ,übertriebene Neigung', , abnorme Leidenschaft‘

14 Einige Lexeme können sowohl eine fachsprachliche als auch eine gemeinsprachliche Verwendung haben, wie z. B. megalomania. 
wie in anglomania, grafomania und italomania festgestellt werden, die auch eine ironisch-kritische Grundhaltung bezeugt.

Von den Kombinationen sind 109 Ad-hoc-Bildungen, die entweder Personennamen (Kyengemania, Brunetta-mania, Michaelmania), Orte (Dubaimania), FestivitätenNamen (Halloween-mania), Ereignisse (Sanremomania), ${ }^{15}$ Fußballvereine (Napolimania, Palermomania), Gegenstände (consolemania, ebookmania), Aktivitäten (shoppingmania, leggeremania, divemania) und Völkernamen (liguro-mania) als erstes Glied haben. Mit solchen Bildungen wird eine besondere Vorliebe für das mit der Erstkonstituenten Bezeichnete ausgedrückt.

Metasprachliche Äußerungen sind auch vertreten und in den wikipedia-spezifischen mania $_{\mathrm{GS}}$-Kombinationen wie grafomania (13), italomania (3), teuto-grafomania (1), anglomania (1) und wiknippomania (1) enthalten. Metadiskursive Äußerungen sind ebenfalls zu finden, wie in cancellomania (9) und templatemania (2):

(3) Negli ultimi tempi c'è una certa cancellomania su wiki. (Wikipedia 2009: Benutzerdiskussion zu Paolo Bertinetto $)^{16}$

(4) Trovo che sia stata un grave superficialità chiamarla templatemania e esagerazione. (Wikipedia 2015: Diskussion zu Argomento fantoccio) ${ }^{17}$

Mit der Äußerung in (3) stellt der Autor fest, dass es eine starke Tendenz gibt, Kommentare zu löschen. Kritisch äußert der Autor in (4) mithilfe der mania-Kombination die Tendenz, etwas nur als Templatemania und Übertreibung zu bewerten.

\section{Sprachvergleich und Fazit}

Bei der vorliegenden Fallstudie handelt es sich um eine kontrastiv konzipierte Untersuchung der Konfixe - phob- (D) und -fob- (IT), -man- (D) und -man- (IT) in den WikipediaKorpora, die über COSMAS II des Leibniz-Instituts für Deutsche Sprache verfügbar sind. Die vorliegende Analyse thematisierte morphologisch-strukturelle, textuell-pragmatische sowie semantische Aspekte der betrachteten Komposita mit den Konfixderivaten -manie/ -mania sowie -phobie/-fobia. Innovatives Moment der Studie ist, dass gegenüber früheren Studien nicht Zeitungstexte als Datenbasis, sondern Texte der internetbasierten Kommunikation - konkret die (Benutzer-)Diskussionsseiten der Online-Enzyklopädie Wikipedia - als Datenmaterial herangezogen werden, deren Sprachmaterial als Produkt interaktionsorientierten Schreibens verstanden werden kann. Die im Kapitel 3 beschriebenen Korpusergebnisse belegen, dass neben medizinfachsprachlichen auch gemeinsprachliche Kombinationen mit den beiden Konfixderivaten in der internetbasierten Kommunikation der Wikipedia vorkommen. Die Fallstudie liefert somit neue Erkenntnisse zum Gebrauchsradius der fokussierten Wortbildungsprodukte.

Die beiden ursprünglich aus der Medizinfachsprache stammenden Konfixe werden sowohl in der deutschen als auch in der italienischen Sprache benutzt und sind in der Gemeinsprache hochproduktiv. Für beide Konfixderivate ist die Zahl der gemeinsprachlichen Kombinationen gegenüber der Zahl der medizinfachsprachlichen Kombinationen höher.

15 Sanremo ist ein bekanntes Musikfestival im italienischen Fernsehen.

16 Übersetzung von CF: In letzter Zeit gibt es in Wikipedia eine gewisse Löschmanie.

17 Übersetzung von CF: Ich denke, dass es wirklich oberflächlich ist, sie Templatemanie und Übertreibung zu nennen. 
Aus einer morphologisch-strukturellen Perspektive kann festgestellt werden, dass die Zahl der Kombinationsmöglichkeiten der Konfixderivate im Deutschen höher ist als im Italienischen. Dies mag auch daran liegen, dass die Bildung neoklassischer Komposita eine Besonderheit im Italienischen ist und nicht zu den sonst üblichen Wortbildungsstrategien zählt. Besonders groß ist die strukturelle Vielfalt im Deutschen bei den * manie $_{\mathrm{GS}}{ }^{-}$ Kombinationen, die in manchen Belegen mit bis zu 20 Gliedern kombiniert werden, die z. T. Hauptsatz-Nebensatz-Konstruktionen entsprechen, was den z. T. ironischen Kontexten, in denen diese Kombinationen zu finden sind, auf einer strukturellen Ebene sprachspielerisch Rechnung trägt. Insbesondere diese sehr komplexen Ad-Hoc-Bildungen können als Besonderheit des interaktionsorientierten Schreibens verstanden werden, was mit folgender Feststellung erläutert werden kann: „Charakteristisch für viele Produkte des interaktionsorientierten Schreibens ist es, dass sie nur für das Funktionieren in der aktuellen Situation konzipiert sind“ (Storrer 2013, S. 337). Eine Vielzahl der analysierten Wortbildungsprodukte ist nämlich hochgradig situativ bedingt und interpretationsbedürftig.

Aus einer semantischen Perspektive ist für die Kombinationen mit -phobie/-fobia eine Verschiebung der Bedeutung auf die Seme ,krankhaft', ,übertriebene Neigung' und ,abnorme Leidenschaft' festzustellen. In beiden Sprachen sind sowohl metadiskursive als auch metasprachliche Äußerungen in der Wikipedia vorhanden. Bei -phobie/-fobia sind die Bedeutungsmerkmale ,Angst ${ }^{`}$ wie auch ,Abneigung' in den analysierten Belegen der Wikipedia vorhanden.

Aus einer textuell-pragmatischen Perspektive ist festzustellen, dass es in beiden Sprachen Belege gibt, die die Funktion haben, Aspekte der kollaborativen Textproduktion metakommunikativ bzw. metasprachlich zu thematisieren (wie dies etwa auch mit itis ${ }_{\mathrm{GS}}$-Kombinationen möglich ist, vgl. Gredel 2018). In der deutschen Sprachversion der Wikipedia ist die Zahl dieser Wortbildungsprodukte jedoch sehr viel höher. In vielen Fällen dienen die gemeinsprachlichen und z.T. wikipedia-spezifischen Wortbildungsprodukte der Normierung von Handlungen in der kollaborativen Textproduktion. Dieses bereits in Korpusbelegen der Korpora wui15 und wdi15 rekonstruierbare Funktionspotenzial der mania-/ fobia-Kombinationen im Italienischen sollte in kommenden Studien mit aktuelleren Korpusdaten weiter betrachtet werden.

Deutlich wurde, dass die als „Europäismen“ einzustufenden Konfixe in der internetbasierten Kommunikation der Wikipedia hochproduktiv sind und sich im multilingualen Kontext der vielsprachigen Wikipedia auch für die Normierung der kollaborativen Textproduktion eignen. Trunkwalter liefert eine plausible Erklärung für den internationalen Einsatz für das Konfix -phob-:

Das Wissen, dass das Konfix -phob- sprachübergreifend produktiv ist, ermöglicht seinen schnellen und sicheren Gebrauch innerhalb internationaler Kommunikation. Geeignete Übersetzungsvarianten müssen nicht gesucht werden, sondern das Konfix -phob- kann unter Anpassung an das jeweilige Sprachsystem in Ad-hoc-Bildungen verwendet werden. (Trunkwalter 2009, S. 300)

Dies ist sicherlich ein Grund dafür, dass die beiden analysierten Konfixe mit ihren Derivaten und zugehörigen Komposita frequent in der internetbasierten Kommunikation der multilingualen Wikipedia auszumachen sind und sich vor allem auch in der Werkstattsprache der Wikipedia wiederfinden. Bemerkenswert ist aus einer morphologisch-strukturellen Perspektive, dass diese Motivationslage der Übernahme so stark ist, dass auch die im Italienischen sonst wenig gebräuchliche Wortbildungsstrategie der neoklassischen Kompositabildung übernommen wird. 


\section{Eva Gredel/Carolina Flinz}

\section{Literatur und Quellen}

Bombi, Raffaella (1993): Riflessioni sulla composizione con affissoidi. In: Incontri Linguistici 15, S. 75-92.

COSMAS II = Corpus Search, Management and Analysis System. Das Portal für die Korpusrecherche in den Textkorpora des Leibniz-Instituts für Deutsche Sprache. Mannheim: Leibniz-Institut für Deutsche Sprache. https://cosmas2.ids-mannheim.de/cosmas2-web (Stand: 21.2.2020).

Dardano, Maurizio (1978): La formazione delle parole nell'italiano di oggi (Primi materiali e proposte). (= Biblioteca di cultura 148). Rom: Bulzoni.

Dardano, Maurizio (1982): Nuovissimo Dizionario della Lingua Italiana. Bd. 1 (a-mi). Rom: Armando Curcio.

De Mauro, Tullio (1999): Grande Dizionario Italiano dell'Uso (GDI). Bd. III: Fm-Man. Turin: UTET.

Devoto, Giacomo/Oli, Gian Carlo (2003): Il Dizionario della Lingua Italiana. Mailand: Le Monnier.

DISC (1997) = Sabatini, Francesco/Coletti, Vittorio (1997): Dizionario Italiano Sabatini Coletti. Florenz: Giunti.

Donalies, Elke (2009): Stiefliches Geofaszintainment - Über Konfixtheorien. In: Müller (Hg.), S. 41-64.

Duden (2016): Der Duden in 12 Bänden. Bd. 4: Die Grammatik: Unentbehrlich für richtiges Deutsch. 9., vollst. überarb. und aktual. Aufl. Berlin: Dudenverlag.

Feine, Angelika (2009): Das Konfix -man-. In: Müller (Hg.), S. 317-350.

Feine, Angelika/Habermann, Mechthild (2005): Eine korpusgestützte Analyse zur deutschen Fremd-Wortbildung. Möglichkeiten und Grenzen. In: Schwitalla, Johannes/Wegstein, Werner (Hg.): Korpuslinguistik deutsch: synchron - diachron - kontrastiv. Würzburger Kolloquium 2003. Tübingen: Niemeyer, S. 89-98.

Gaeta, Livio/Ricca, Davide (2006): Productivity in Italian word formation: A variable-corpus approach. In: Linguistics 44, 1, S. 57-89.

Gaeta, Livio/Ricca, Davide (2015): Productivity. In: Müller, Peter O./Ohnheiser, Ingeborg/Olsen, Susan/ Rainer, Franz (Hg.) (2015): Word-Formation. An International Handbook of the Languages of Europe. Bd. 2. (= Handbücher zur Sprach- und Kommunikationswissenschaft (HSK) 40.2). Berlin/Boston: De Gruyter Mouton, S. 842-858.

Gredel, Eva (2018): Itis-Kombinatorik auf den Diskussionsseiten der Wikipedia: Ein Wortbildungsmuster zur diskursiven Normierung in der kollaborativen Wissenskonstruktion. In: Zeitschrift für Angewandte Linguistik 68, 1, S. 35-72.

Iacobini, Claudio (2004): Composizione con elementi neoclassici. In: Grossmann, Maria/Rainer, Franz (Hg.): La formazione delle parole in italiano. Tübingen: Niemeyer, S. 69-96.

Iacobini, Claudio (2015): Foreign word-formation in Italian. In: Müller, Peter O./Ohnheiser, Ingeborg/ Olsen, Susan/Rainer, Franz (Hg.) (2015): Word-Formation. An International Handbook of the Languages of Europe. Bd. 3. (= Handbücher zur Sprach- und Kommunikationswissenschaft (HSK) 40.3). Berlin/ Boston: De Gruyter Mouton, S. 1660-1678.

Il Grande Dizionario Garzanti della Lingua Italiana (1987): Mailand: Garzanti.

Kirkness, Alan (2001): Europäismen/Internationalismen im heutigen deutschen Wortschatz. Eine lexikographische Pilotstudie. In: Stickel, Gerhard (Hg.): Neues und Fremdes im deutschen Wortschatz: Aktueller lexikalischer Wandel. (= Jahrbuch des Instituts für Deutsche Sprache 2000). Berlin/New York: De Gruyter, S. 105-130.

Lüngen, Harald/Kupietz, Mark (2017): CMC Corpora in DeREKo. In: Bański, Piotr/Kupietz, Marc/Lüngen, Harald/Rayson, Paul/Biber, Hanno/Breiteneder, Evelyn/Clematide, Simon/Mariani, John/Stevenson, Mark/Sick, Theresa (Hg.): Proceedings of the Workshop on Challenges in the Management of Large Corpora and Big Data and Natural Language Processing (CMLC-5+BigNLP) 2017 including the papers 
from the Web-as-Corpus (WAC-XI) guest section. Birmingham, 24 July 2017, Mannheim: Institut für Deutsche Sprache, S. 20-24.

Margaretha, Eliza/Lüngen, Harald (2014): Building Linguistic Corpora from Wikipedia Articles and Discussions. In: Beißwenger, Michael/Oostdijk, Nelleke/Storrer, Angelika/van den Heuvel, Henk (Hg.): Building and Annotating Corpora of Computer-mediated Communication: Issues and Challenges at the Interface between computational and corpus linguistics. (= Journal for Language Technology and Computational Linguistics (JLCL) 29.2). Regensburg: GSCL, S. 59-82.

Martinet, Andrè (1988): Sintassi generale. (= Biblioteca universale Laterza 253). Rom/Bari: Laterza.

Migliorini, Bruno (1963): I prefissoidi (il tipo aereomobile, radiodiffusione). In: Migliorini, Bruno (Hg.): Saggi sulla lingua del Novecento. 3. rev. Aufl. (= Biblioteca di lingua nostra 1). Florenz: Sansoni, S. 9-60.

Müller, Peter O. (Hg.) (2009): Studien zur Fremdwortbildung. (= Germanistische Linguistik 197-198). Hildesheim u. a.: Olms.

Scalise, Sergio (1983): Morfologia lessicale. Padua: CLESP.

Serianni, Luca (1988): Grammatica italiana: italiano comune e lingua letteraria: suoni, forme, costrutti. Turin: UTET.

Storrer, Angelika (2013): Sprachstil und Sprachvariation in sozialen Netzwerken. In: Frank-Job, Barbara/ Mehler, Alexander/Sutter, Tilmann (Hg.): Die Dynamik sozialer und sprachlicher Netzwerke. Wiesbaden: Springer VS, S. 331-366.

Tekavčić, Pavao (1980): Grammatica storica dell'italiano. III. Lessico. Bologna: Il Mulino.

Trunkwalter, Julia (2009): Das Konfix - phob-. Ein Überblick über Etymologie, Morphologie, Semantik und Pragmatik der Wortbildungseinheit -phob-. In: Müller (Hg.), S. 261-316.

Wikipedia (2004): Diskussion zum Lemma Funzione die ripartizione della variabile casuale normale. https:// it.wikipedia.org/wiki/Discussione:Funzione_di_ripartizione_della_variabile_casuale_normale (Stand: 14.7.2020).

Wikipedia (2007): Benutzerdiskussion zu Paolo Bertinetto. https://it.wikipedia.org/wiki/Discussioni_utente: Paolo_Bertinetto (Stand: 14.7.2020).

Wikipedia (2009): Diskussion zum Lemma Waffenfarbe. https://de.wikipedia.org/wiki/Diskussion: Waffenfarbe (Stand: 14.7.2020).

Wikipedia (2013): Benutzerdiskussion zu Riccardo Rovinetti. https://it.wikipedia.org/wiki/Discussioni_ utente:Riccardo_Rovinetti (Stand: 14.7.2020).

Wikipedia (2014): Diskussion zum Lemma Nexialismus. https://de.wikipedia.org/wiki/Diskussion: Nexialismus (Stand: 14.7.2020).

Wikipedia (2015): Diskussion zu Argomento fantoccio. https://it.wikipedia.org/wiki/Discussione:Argomento_ fantoccio (Stand: 14.7.2020).

Dr. Eva Gredel (B. Sc.)

Universität Mannheim

Seminar für deutsche Philologie

Germanistische Linguistik

Schloss, Ehrenhof West, Raum 253

68131 Mannheim

E-Mail: eva.gredel@phil.uni-mannheim.de
Dr. habil. Carolina Flinz

Università degli Studi di Milano

Dipartimento di Scienze della Mediazione Linguistica

e di Studi Interculturali

Piazza Indro Montanelli, 1

20099 Sesto San Giovanni (MI)

Italien

E-Mail: carolina.flinz@unimi.it 